\title{
Frontières
}

\section{DANON-BOILEAU, Henri, De la vieillesse à la mort, point de vue d'un usager, Paris, Calmann-Lévy, 2000, 216 p.}

\section{Denise Badeau}

Volume 16, numéro 1, automne 2003

URI : https://id.erudit.org/iderudit/1073772ar

DOI : https://doi.org/10.7202/1073772ar

Aller au sommaire du numéro

Éditeur(s)

Université du Québec à Montréal

\section{ISSN}

1180-3479 (imprimé)

1916-0976 (numérique)

Découvrir la revue

Citer ce compte rendu

Badeau, D. (2003). Compte rendu de [DANON-BOILEAU, Henri, De la vieillesse à la mort, point de vue d'un usager, Paris, Calmann-Lévy, 2000, 216 p.] Frontières, 16(1), 99-100. https://doi.org/10.7202/1073772ar d'utilisation que vous pouvez consulter en ligne.

https://apropos.erudit.org/fr/usagers/politique-dutilisation/ 
chanalytique " car, de fait, l'auteur nous entretient surtout de ce sujet. Ceci étant dit, l'ouvrage peut être investi d'une double crédibilité, car son auteur est à la fois âgé et thérapeute. À ce titre, il a eu l'occasion de recueillir plus d'un témoignage de patients âgés sur le sujet.

Les buts énoncés sont: "de démêler en quoi le point de vue psychanalytique, alimenté par l'effort d'auto-analyse du vieillard que je suis, permettrait d'envisager autrement la fin de l'existence » (p. 9) et "de réduire, en les débarrassant d'une part de leur gangue névrotique, certaines des blessures et des angoisses que suscitent le vieillissement et la mort proche» (p. 9). L'auteur se propose de mettre "l'accent sur les aspects toujours vivaces des désirs, conflits et culpabilités inconscientes, sur l'importance du fonctionnement du fantasme de Toute-Puissance et de la pensée " magique » pour laquelle souhaiter, penser, faire, faire arriver sont équivalents» (p. 9).

L'ouvrage se divise en deux parties intitulées réciproquement: Booz endormi ou la sexualité des vieillards et l'art d'accommoder les restes. La première partie traitant de la sexualité des aînés le fait en présentant différents couples : les couples vieux-jeune, «il est plus âgé qu'elle » ou vieille-jeune, "elle est plus âgée que lui ", le couple senior-senior ou le couple ordinaire constitué de personnes hétérosexuelles d'âge semblable, le couple masculin homosexuel vieux-jeune. Cette première partie nous entretient aussi de la masturbation. L'art d'accommoder les restes s'intéresse aux problèmes soulevés par les renoncements nécessaires, s'intéresse également aux activités sublimatoires, aux conditions essentielles du bien vieillir, au comment profiter de la façon la plus satisfaisante possible des autres et de soimême. Elle se termine par quelques paragraphes sur la grand-parentalité. L'auteur souligne le rôle et l'intérêt d'une psychothérapie psychanalytique pour les personnes âgées.

Selon Danon-Boileau, les relations entre vieux et jeunes ne s'accompagnent pas du «non-dit ou du nonconçu " qui accompagne souvent, même trop souvent les relations des couples de vieillards. II est plus âgé qu'elle, cette relation est généralement bien acceptée par l'opinion surtout si monsieur vient d'un milieu socioculturel et financier élevé. Quand la rupture survient, dans la majorité des cas, elle est initiée par la femme qui se lasse de son amantvieillard qu'elle ne peut plus admirer, qui ne la protège plus et de surcroît l'ennuie par son discours répétitif. S'ajoute à cela l'incapacité de son amant de la satisfaire sexuellement. Elle supporte mal ses avances qui parfois la dégoûtent. La rupture porte atteinte à l'image de soi du vieillard. Le retrait de l'amour de sa jeune amante lui apparaît comme irréparable entraînant chez lui honte et culpabilité, dépression parfois grave, détérioration de ses facultés physiques et psychologiques. Quand le vieillard prend l'initiative de la rupture, il le fait parce qu'il perçoit cette rupture comme imminente et qu'il a besoin de maîtriser la situation " par désir narcissique de se protéger, de sauvegarder son image » (p. 33).

Quand elle est plus âgée que lui, « la relation se détériore beaucoup plus rapidement » (p. 37).

Si l'on envisage, même à grands traits, ce qui concerne I'homme au cours d'une liaison de ce type, on note qu'aux satisfactions amoureuses s'ajoutent les plaisirs narcissiques, la fierté de damer le pion aux aînés, aux véritables " ayant-droit » en étant traité sexuellement comme un homme par une « vraie femme » (p. 35).

La force de l'interdit de l'inceste mère-fils expliquerait, selon l'auteur, la fragilité de cette relation. L'abandon de la femme par son jeune amant risque de la précipiter dans le « sentiment de la vieillesse menaçante [...] et dans la dépression" (p. 41). Certains couples, dans les deux cas, trouvent différentes formes d'équilibre ou d'aménagement leur permettant de durer.

Les liaisons masculines homosexuelles vieux-jeune peuvent «évoluer elles aussi dans deux directions principales: la rupture ou l'aménagement » (p. 41). L'adulte âgé joue alors auprès du jeune le rôle de bonne mère, permissive, donnante, le traitant comme il aurait aimé être traité par sa propre mère. L'auteur n'aborde cependant pas les relations féminines homosexuelles.

Ils sont du même âge ou la relation senior-senior: Danon-Boileau affirme n'avoir jamais été consulté par un couple de seniors. De nombreux thérapeutes pourraient faire la même affirmation, les seniors éprouvant une certaine gêne quand ce n'est pas de la honte ou de la culpabilité à se présenter comme encore actifs sexuellement ou désirant l'être. Le senior, quand il ose parler de sa vie sexuelle, insiste sur " un réel désir psychique et l'absence d'érection; les fantasmes habituels semblent persister, mais ne sont suivis $d^{\prime}$ aucun effet [...]» (p. 64). 
L'auteur parle de la disparition progressive du désir jusqu'au « tarissement proprement dit» (p. 64). Chez la femme, la disparition du désir semble "s'intégrer naturellement dans l'ordre des choses » (p. 68). La tendresse prendrait «le relais des élans passionnels ".

[...] in fine, c'est le même refus de vieillir et de mourir chez I'homme et chez la femme, mais il est plus clairement narcissique chez la femme que chez

I'homme. Celui-ci se sent " obligé » de passer par l'acte sexuel, le fantasme de ToutePuissance passant chez lui par l'érection, tandis que chez la femme il semble passer davantage par le besoin de séduire que par le désir d'être pénétrée (p. 69).

Considérée par des tiers, la relation sexuelle vécue par des vieillards est une activité mortifère et la masturbation témoigne d'une transgression. Triste constat d'une évolution qui tarde à se faire quant à la reconnaissance des aînés comme des personnes adultes à part entière. $Y$ a-t-il place pour une évolution sexuelle à I'aube de ce XXI'e siècle?

Concluant cette première partie, l'auteur déplore le manque d'information sur l'évolution physiologique de la sexualité avec l'âge, ce qui permettrait aux individus de s'adapter aux transformations naturelles qui surviennent « en évitant la surcharge d'angoisse liée à l'ignorance » (p. 82) et encouragerait au maintien d'une vie sexuelle active, à leur rythme, et où la tendresse à travers les gestes de la vie quotidienne "comblerait les aléas de l'érotisme » (p. 82).

Dans la deuxième partie de son ouvrage, l'auteur nous invite à tirer le meilleur parti de ce qui reste.

Si toute la vieillesse est un naufrage (selon le mot d'un grand vieillard, vigoureux, créateur jusqu'au bout...), elle $n^{\prime}$ interdit pas en effet d'aborder de temps en temps sur des plages accueillantes : encore faut-il que l'intéressé ait décidé de construire son radeau et de se battre jusqu'à la côte (p. 87).

Il distingue entre résignation et renoncement en ce que la première est une soumission à une force extérieure tandis que le deuxième relève d'une décision personnelle. II aborde la vieillesse source d'espoir et source de renoncements. Source d'espoir: espoir de nouvelles rencontres, d'amitiés riches et fidèles, éventail de possibilités (bénévolat, création, recherche, sublimation "à la carte", espoir d'un au-delà, recours à la religion pour juguler ses craintes).
Source de renoncements : se donner la mort pour éviter la détérioration ou la douleur sans espoir ou pour disposer de sa destinée, perte de proches, de personnes de sa génération.

[...] si le renoncement excessif s'accompagne le plus souvent d'un désir, évident pour d'autres mais complètement inconscient pour l'intéressé, de maîtrise ou de Toute-Puissance, cette composante fait partie de toute forme de renoncement, même le plus favorable. C'est en effet grâce à cette satisfaction narcissique de maîtrise que le renoncement prend son sens complet, sa valeur positive face à la nécessité (p. 103).

L'auteur consacre quelques pages à ce qu'il appelle les « brumes d'hiver ", c'est-à-dire les problèmes d'ordre cognitif: oubli des noms propres, difficultés de concentration et d'apprentissage, ressassement; «[...] nombre de suicides de seniors sont motivés par la prise de conscience des débuts - nets - d'une atteinte intellectuelle, i.e. avant que ne s'émousse le sentiment de soi " (p. 162).

Il insiste sur le rôle de l'entourage des personnes âgées dans leur attitude face à la vieillesse et la fin de la vie: « [...] il n'est pas rare que la mauvaise volonté de l'environnement fasse le lit du découragement et de l'abandon » (p. 186). Il consacre quelques pages à la grand-parentalité qui permet à la personne âgée de se sentir encore bonne, encore introjectable, de transmettre à ses petits-enfants « un bon objet protecteur ; " [ [...] offrir cette image rassurante, c'est encore donner, c'est une façon de redevenir, malgré sa disparition, un bon objet dont la permanence est assurée en tant que telle chez l'autre » (p. 209). Il termine en souhaitant « que quelles que soient les raisons inconscientes qui, dans les derniers instants, sous-tendent le " beau mourir ", l'important est qu'il ait lieu » (p. 209).

Cet ouvrage tout en étant empreint d'un réalisme indéniable concernant la vieillesse invite le lecteur à se donner les moyens de vivre sa sexualité jusqu'au bout de la vie, à inventorier de nouvelles activités, à recourir à un soutien thérapeutique pour redécouvrir le plaisir du faire.

Dirions-nous qu'il a atteint les buts énoncés? En partie seulement. Le lecteur comprend mieux les enjeux d'une relation de couple à l'âge avancé de même que le besoin d'exprimer sa sexualité à son rythme et selon les disponibilités de parte- naires et de fonctions. Est-ce que les angoisses suscitées par le vieillissement et la mort proche sont ou seront réduites? C'est l'expérience qui en témoignera mais un certain scepticisme demeure tant il y a loin entre connaissances, compréhension et changement d'attitudes. La perspective de durer au-delà de la mort et ce à travers la vie biologique, psychologique, intellectuelle donnée est sans doute l'ultime consolation.

Denise Badeau 